EPJ Web of Conferences 57, 04001 (2013)

DOI: $10.1051 /$ epjconf/20135704001

(C) Owned by the authors, published by EDP Sciences, 2013

\title{
Ultrafast AMO physics at the LCLS x-ray FEL
}

\author{
P.H. Bucksbaum and J.M. Glownia \\ Stanford PULSE Institute, Stanford University and SLAC National Accelerator Laboratory, \\ Menlo Park, CA 94025, USA
}

\begin{abstract}
The Linac Coherent Light Source at the SLAC National Accelerator Laboratory, Stanford University, began operation in 2009 as the world's first hard x-ray free electron laser. Early experiments have concentrated on atomic physics, and have demonstrated several key features of the ultrafast high field $\mathrm{x}$-ray-atom interaction. This paper reviews some of these early results.
\end{abstract}

\section{INTRODUCTION: WORLD'S BRIGHTEST ULTRAFAST X-RAY SOURCE, LCLS}

The new class of so-called "fourth generation" synchrotron light sources are a revolutionary departure from storage ring-based electron synchrotrons. They are true x-ray lasers, which use relativistic electrons as a gain medium. The Linac Coherent Light Source (LCLS) at the SLAC National Accelerator Laboratory in Menlo Park California is the world's first hard x-ray free electron laser (XFEL) [1]. It produces coherent $\mathrm{x}$-ray fields that exceed the brightness of any previous source (measured in energy per unit time per unit solid angle per unit frequency interval) by a factor of one billion or more. The increase of peak brightness is due to a combination of increased pulses energy and decreased pulse duration.

These novel lasers allow us to observe the fastest timescales in atoms and molecules, which are due to the interactions among electrons. The timescales involved can be characterized by electronic energy separations, which can be many electron volts. Any ultrafast laser source is limited to pulse durations no shorter than a single cycle of the electromagnetic field. For the conventional Ti : Sapphire laser $(\lambda=800 \mathrm{~nm})$, this is about $2 \mathrm{fs}$. But for $\mathrm{x}$-rays, the pulse duration can be much shorter. Simulations show that the LCLS is capable of sub-femtosecond pulses, which could capture electronic coherences in molecules. X-rays can reveal multielectron processes inside atoms and molecules.

Furthermore, these sources have not yet reached their intrinsic limits in pulse duration, where sub-femtosecond pulses are possible [2]. Accelerator and x-ray laser scientists and engineers at SLAC have also begun to explore techniques that will one day provide routine operation with pulses whose time-bandwidth product is near the Fourier transform limit [3]. Such control over $\mathrm{x}$-ray fields could be exploited just as in the field of quantum optics with visible and ultraviolet laser pulses.

This short review will highlight some of the first results, and the opportunities for further work in strong-field x-ray physics of atoms and molecules. The work described here was all done at the LCLS $\mathrm{x}$-ray free electron laser by the Stanford PULSE Institute AMO group in collaboration with other AMO research groups around the world [4-9]. Some of the experiments described form the thesis research of two Stanford students, Mike Glownia and James Cryan [10, 11].

This is an Open Access article distributed under the terms of the Creative Commons Attribution License 2.0, which permits unrestricted use, distribution, and reproduction in any medium, provided the original work is properly cited. 

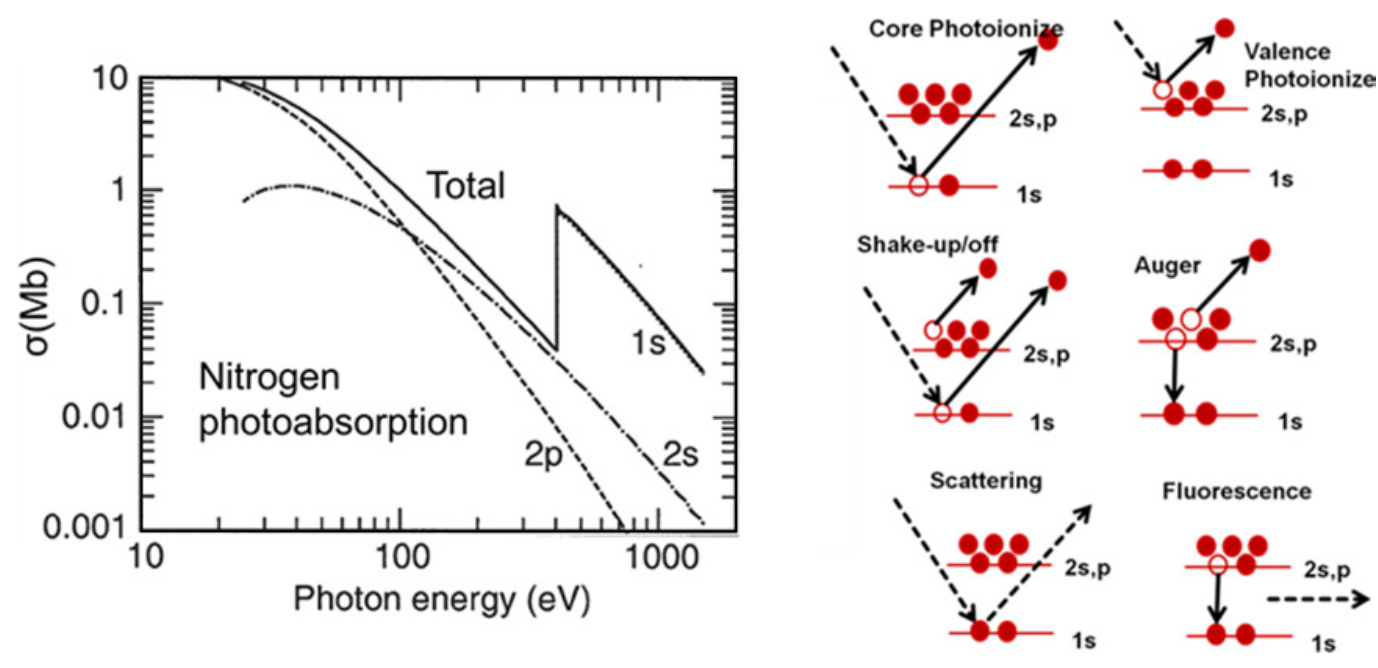

Figure 1. Left: Nitrogen absorption cross sections (After Yeh and Lindau [12]). Right: Schematic representation of the various $\mathrm{x}$-ray atom interactions in nitrogen described in the text.

\section{X-RAY INTERACTIONS WITH ATOMS}

$\mathrm{X}$-rays interact with multi-electron atoms and molecules in a fundamentally different way than optical radiation because the interaction cross sections favour the inner electrons, which are transparent at visible wavelengths.

The basic processes of $\mathrm{x}$-ray laser interactions are shown in Figure 1. The graph on the left shows the partial photoabsorption cross section for each of the occupied shells. Despite the presence of five electrons in the $n=2$ shells of nitrogen, the total absorption for x-ray energies above the "k-edge" at $410 \mathrm{eV}$ is dominated by photoionization of the two $n=1$ electrons in the k-shell of the atom.

The six diagrams on the right show the various processes of the x-ray-atom interaction. The top left process, core photoionization, dominates the valence photoionization shown in the top right diagram for x-ray energies higher than the $1 \mathrm{~s}$ binding energy of $410 \mathrm{eV}$. Sometimes the photoemission is accompanied by inelastic scattering which can leave the valence electron in an excited state (shakeup) or the continuum (shake-off). Elastic scattering may also occur, although the inelastic cross section dominates at the soft $\mathrm{x}$-ray energies used in the AMO experiments at LCLS. The remaining two diagrams show the two relaxation processes following the creation of a core vacancy. By far the dominant process is Auger relaxation, in which two valence electrons exchange energy, driving one of them into the core hole while the other is ejected into the continuum. This is a non-radiative process, which is essentially quantum evaporative cooling by the electrons. The lifetime for Auger relaxation depends on electronelectron correlation, and in particular on the overlap between the valence and core electrons. The lifetime is typically on the order of a few femtoseconds. Fluorescence is also possible, although this process is much slower for low-Z atoms such as nitrogen, on the order of hundreds of femtoseconds to picoseconds.

For weak x-ray sources such as x-ray tubes, or even third generation synchrotrons, Figure 1 is a fair representation of the processes we will expect to excite in molecular nitrogen in the presence of $\mathrm{x}$-rays. However, an X-ray laser source can be bright enough for photoabsorption rates to compete with Auger rates. The saturation fluence $f_{\text {sat }}=1 / \sigma_{a b s} \approx 10^{18} \mathrm{~cm}^{-2}$ describes the total fluence (photons per unit area) of x-rays required to excite a transition with high probability for a typical cross section of one megabarn (Mb). In fact, as we shall show, the photon fluence available is far more than this extending as high as $10^{21} \mathrm{~cm}^{-2}$ in a focused LCLS X-ray pulse. Therefore a number of nonlinear absorption processes dominate the physics of the interaction. 
Table 1. LCLS properties vs. synchrotron x-ray sources.

\begin{tabular}{|l|l|l|}
\hline & LCLS & Synchrotrons \\
\hline Strong fields & $10-100$ atomic units & 0.001 atomic unit \\
\hline Ultrafast pulses & $5-200 \mathrm{fs}$ & $50 \mathrm{ps}$ \\
\hline High single pulse & $10 \mathrm{~kJ} / \mathrm{cm}^{2} \approx$, & $10 \mathrm{~mJ} / \mathrm{cm}^{2} \approx$ \\
fluence & $10^{20} \mathrm{~cm}^{-2} @ 1 \mathrm{keV}$ & $10^{14} \mathrm{~cm}^{-2} @ 1 \mathrm{keV}$ \\
\hline Coherence & Transverse: TEM00 & Incoherent \\
& Longitudinal: $\sim 1 \mathrm{eV}$ & \\
\hline
\end{tabular}

\section{FELs: ELECTRON STIMULATED EMISSION}

The gain medium in an x-ray free electron laser is a dense cloud of relativistic electrons, which emit synchrotron radiation as they pass through an array of alternating magnetic fields in a structure called an undulator. At LCLS the fields are formed by permanent magnets, with an alternating period of $3 \mathrm{~cm}$. The electrons are highly relativistic, typically on the order of $1-15 \mathrm{GeV}$, so that the relativistic $\gamma$ factor ranges as high as 30,000 .

The electrons are traveling only a part in a billion slower than the speed of light, so that they would travel right along with their radiation were it not for the fact that the electrons wiggle due to the oscillating magnetic fields, while the light travels in a straight line. This produces a phase slip between the electrons and their own synchrotron emission. Synchrotron fields whose wavelength is just equal to the differential progress of the electrons and the x-rays over one full period of magnetic field alternation are in resonance for constructive interference of emission as the electrons traverse the undulators. The resonance condition is given by

$$
\lambda_{r}=\frac{\lambda_{a}}{2 \gamma^{2}}\left(1+\frac{K^{2}}{2}+\gamma^{2} \theta^{2}\right)
$$

where $\lambda_{r}$ is the resonant $\mathrm{x}$-ray wavelength, $\lambda_{a}$ is the undulator period, $K$ is the product of the maximum angle of deviation of the oscillating path of the electrons in the lab frame times the relativistic $\gamma$ factor, the electron energy is $\gamma m c^{2}$, and $\theta$ is the angle between the radiation and the undulator axis.

As this resonant radiation builds, it feeds back on the electrons by accelerating those whose wiggling motion is in phase with the synchrotron field, and decelerating those whose motion is out of phase. In this way the electrons begin to bunch together. The bunching is on the micron scale in the rest frame of the electrons, but it is on the Angstrom scale in the laboratory frame of the undulator magnets. The microbunched electrons all radiate in phase, producing the x-ray laser radiation of the FEL.

As we have stated, radiation produced by LCLS is approximately one billion times brighter than the radiation from the brightest third generation synchrotrons, such as the Advanced Photon Source at Argonne National Laboratory. About six orders of magnitude of this increase is simply due to the high energy per pulse compared to a synchrotron. LCLS typically produces on the order of one millijoule of energy on every shot. The other three orders of magnitude in increased brightness is due to the short pulse duration of the x-ray FEL. This is typically on the order of $100 \mathrm{fs}$, vs. 100 ps for a synchrotron. A general comparison is shown in Table 1.

\section{STRONG FIELD X-RAY-ATOM INTERACTIONS}

Table 1 illustrates how LCLS is the first true strong field ultrafast x-ray source, and it brings strong field physics within reach at $\mathrm{x}$-ray wavelengths for the first time. This new physics has been explored in several experimental studies carried out during the first three years of operation of LCLS. In this brief review, we will concentrate on some of the most basic studies, which were carried out by collaborations 
of several groups at Stanford, Argonne National Laboratory, Western Michigan University, and the Ohio State University.

\subsection{Rapid "sequential" ionization}

In Section 2 we showed how the available fluence of x-ray photons from a single tightly focused pulse of LCLS can greatly exceed the saturation fluence for k-shell photoemission. We therefore expect to see very high order multiple ionization, as the k-shell is ionized as rapidly as Auger relaxation can refill it. This was the first strong-field process observed using LCLS, in experiments led by the Young group from Argonne National Laboratory [8]. The LCLS beam focused to a few square microns was able to strip all of the electrons from $\mathrm{Ne}$ atoms, provided the photon energy was sufficient for 1-photon ionization of the $1 \mathrm{~s}$ shell at each charge stage, i.e. a photon energy greater than $1.36 \mathrm{keV}$, the energy required to ionize $\mathrm{Ne}^{9+}$. The interpretation of this result is that the likely process is sequential $\mathrm{k}$-shell ionization $(\gamma)$, followed by rapid Auger relaxation (A) at every step:

$$
N e \stackrel{\gamma}{\rightarrow} N e^{+} \stackrel{A}{\rightarrow} N e^{2+} \stackrel{\gamma}{\rightarrow} N e^{3+} \stackrel{A}{\rightarrow} N e^{4+} \stackrel{\gamma}{\rightarrow} N e^{5+} \stackrel{A}{\rightarrow} N e^{6+} \stackrel{\gamma}{\rightarrow} N e^{7+} \stackrel{A}{\rightarrow} N e^{8+} \stackrel{\gamma}{\rightarrow} N e^{9+} \stackrel{\gamma}{\rightarrow} N e^{10+} .
$$

Note that here the symbol $\gamma$ refers to a photoionization event. In this way it can be seen that six photons are required to completely strip a Ne atom down to the bare nucleus. Note that in the last step Auger is no longer possible, since it requires at least two electrons in the $\mathrm{n}=2$ shell.

This " $\gamma A \gamma A$ " process described above requires that the Auger relaxation rate is very fast compared to the single photon ionization rate to keep the sequence in order. Auger rates depend on the Coulomb interaction between the valence electrons that participate. In Ne atoms, these rates are on the order of a few femtoseconds. Auger rates do not vary much with atomic species, since most atoms are about the same size and therefore electron-electron repulsion in the valence shells is not strongly dependent on atomic number, so long as there are at least two electrons in the valence shell. However, the competing process of photoionization can be very fast indeed at LCLS. As we have seen, the photon fluence in a single $100 \mathrm{fs}$ pulse may exceed the saturation fluence for photoionization by a factor of 100, which means that the ionization rate can be as short as $1 \mathrm{fs}$. This creates the opportunity for rapid sequential double ionization without Auger relaxation:

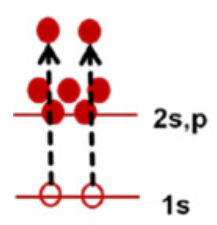

$$
\mathrm{Ne} \stackrel{\gamma}{\rightarrow} \mathrm{N} e^{+} \stackrel{\gamma}{\rightarrow} \mathrm{Ne} e^{2+}
$$

Figure 2. Two-photon single-site double core hole formation.

Note that two sequential absorptions without relaxation deplete the 1s shell completely, which reduces the absorption cross section dramatically, rendering the atom relatively transparent to x-rays until Auger relaxation refills a 1 s orbital.

One of the first LCLS experiments searched for this exotic " $\gamma \gamma A$ " process in nitrogen molecules placed in a tighly focused LCLS beam, during an experimental run in November 2009. The collaboration was led by Nora Berrah of Western Michigan University [6, 7, 13]. Several data sets were collected with the same total fluence but different pulse duration. As the pulses are shortened for the same total fluence, the photon intensity increases, and therefore we expect the rate of photoionization to also increase. To enhance this intensity-dependent effect as much as possible we added an additional data set at the shortest pulse duration we could extract from the LCLS during a second run led by Ryan Coffee of 


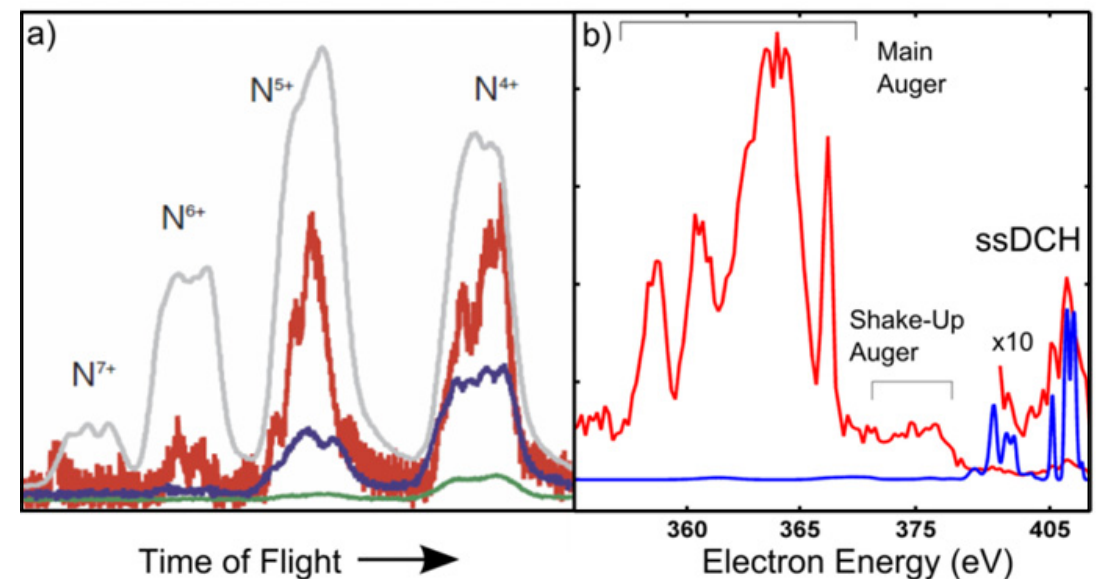

Figure 3. a) Nitrogen charge state distributions following intense x-ray ionization, demonstrating the phenomenon of frustrated ionization. The red, blue, and green curves all have pulse energies of approximately $0.25 \mathrm{~mJ}$, and pulse durations of $280 \mathrm{fs}, 80 \mathrm{fs}$, and 5-10 fs, respectively. The nitrogen molecules become relatively transparent to $\mathrm{X}$-rays when both $1 \mathrm{~s}$ electrons have been ionized faster than Auger relaxation can refill them. Therefore shorter pulses cannot strip as many electrons from the atoms as longer pulses with the same photon fluence. The grey curve is a longer pulse with $2.2 \mathrm{~mJ}$ to show the reference spectrum. (Adapted from Hoener et al. [7]). b) Auger electron spectra obtained using the shortest $\mathrm{x}$-ray pulses. The large feature spanning energies of $355 \mathrm{eV}$ to $365 \mathrm{eV}$ correspond to normal Auger following creationg of a single core vacancy. The single-site double core vacancy Auger spectrum is centered on $405 \mathrm{eV}$. The blue curve is a calculation (adapted from Cryan, et al. [4]).

the SLAC/Stanford group [4]. The resulting time-of-flight spectra are shown in Figure 3. The longest pulses are capable of removing all of the electrons from a nitrogen atom or a nitrogen molecule, but the shortest pulses cannot, because the Auger rate is to slow compared to the ionization rate. Analysis shows that the onset of this saturation transparency is what we expect from depletion of the 1s shell by rapid $\mathrm{x}$-ray ionization $[7,8]$.

\subsection{Auger spectroscopy of "hollow molecules"}

The observation of frustrated ionization indicates the presence of a new state of matter, the "hollow molecule" where the core electrons are absent. We studied the properties of this state by performing Auger spectroscopy on hollow molecules. This is an entirely new way to study femtosecond chemical dynamics with Auger electrons that probe the local valence structure of molecules near a specific atomic core.

We employed $1.1 \mathrm{keV}$ photons, which is well above the threshold for k-shell ionization in nitrogen. The most distinct features arise from sequential $\mathrm{x}$-ray ionization of the same atom in the molecule, which we called "single site" double core vacancies to differentiate them from double ionization involving one k-electron from each atom in the diatomic molecule. We observed a multi-peaked single-site double core vacancy Auger electron spectrum near $413 \mathrm{eV}$, which agreed well with ab initio calculations.

\subsection{Ultrafast pump-probe experiments utilizing LCLS}

Angle-dependent Auger emission can help reveal more features of hollow molecules, but gas phase molecular spectroscopy generally cannot yield orientation-dependent information because the molecules are randomly oriented in the gas. We addressed this problem by aligning the molecules in advance, using a second laser in a "pump-probe" arrangement. 
a)

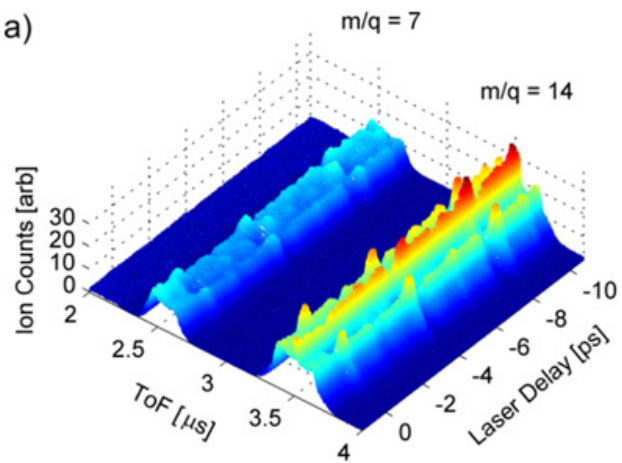

b)

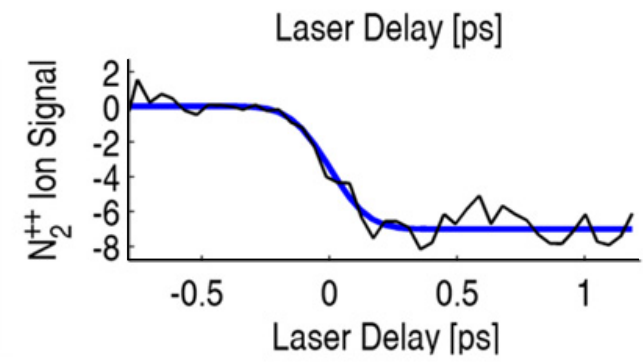

Figure 4. Periodic quantum alignement of nitrogen molecules are viewed here through their dissociation. a) Pumpprobe time-delay dependent ion time-of-flight (ToF) spectra of molecular nitrogen. The two ridges correspond to the ToF spectrum near $\mathrm{m} / \mathrm{q}=14$ at $3.5 \mu \mathrm{s}$, showing $\mathrm{N}^{+}$and $\mathrm{N}_{2}^{++}$fragments; and $\mathrm{m} / \mathrm{q}=7$, at $2.5 \mu \mathrm{s}$, which shows the $\mathrm{N}^{++}$fragments. The width of these features is due to the dissociation kinetic energy of the atomic fragments. Negative delay indicates that the optical pulse precedes the $\mathrm{x}$-ray pulse. Periodic rotational alignment features are clearly visible in all peak structures. b) A lineout at the position of the molecular dication $\mathrm{N}_{2}^{++}$, which shows how it becomes depleted if the $\mathrm{x}$-rays precede the laser field. This is due to laser-induced dissociation of the dications formed by the x-rays [5].

Pump-probe spectroscopy consists of performing a series of experiments in which the dynamics under investigation is initiated by a short laser pulse, and then after a variable delay the state of the system is probed by a second pulse. The experimental results are then viewed sequentially according to increasing delay, producing a stroboscopic movie of the dynamics. Pump-probe spectroscopy works well when both pulses are derived from the same laser source, because then the delays can be set up precisely by varying the relative path length of the laser beam for the pump vs. probe. At LCLS this is extremely challenging because the pump and probe are produced in two entirely different lasers. The method used for pump probe spectroscopy locks the optical laser to the same radio frequency reference that is used to drive the linear accelerator.

We performed the first pump-probe experiments at LCLS to align nitrogen molecules for angleresolved Auger spectroscopy [4, 5, 9]. The transient signal we chose to investigate was the periodic rotational alignment of nitrogen gas that had been subject to an impulse with an intense linearly polarized ultrafast $800 \mathrm{~nm}$ laser. The molecules undergo transient stimulated rotational Raman scattering, which performs a unitary transformation of each $\mid J, m>$ state into a broad coherent superposition of $m$ states with many different $J$ 's The energy eigenvalues $E$ of the rotational states are proportional to $E_{0} J(J+1)$, and since they are all integer multiples of $E_{0}$, they rephase periodically. In nitrogen this quantum revival period is $5 \mathrm{ps}$.

Our time-resolved x-ray/optical pump-probe experiments at LCLS showed that the natural timing jitter between the x-ray laser and a local laboratory laser could be improved substantially by employing both feedback methods to counteract drift, and post-analysis binning techniques to synchronize the two pulses.

Transient molecular nitrogen alignment revival features were resolved in time-dependent $\mathrm{x}$-ray-induced fragmentation spectra, as shown in Figure 4. These alignment features were used to find the temporal overlap of the pump and probe pulses.

The strong-field dissociation of x-ray generated quasi-bound molecular dications $\mathrm{N}_{2}^{++}$was used to establish the residual timing jitter. This analysis shows that the relative arrival time of the Ti : Sapphire laser and the x-ray pulses had a distribution with a standard deviation of approximately $120 \mathrm{fs}$. The largest contribution to the jitter noise spectrum was the locking of the laser oscillator to the reference $\mathrm{RF}$ of the accelerator. later improvements have now reduced the jitter to better than $50 \mathrm{fs}$. 

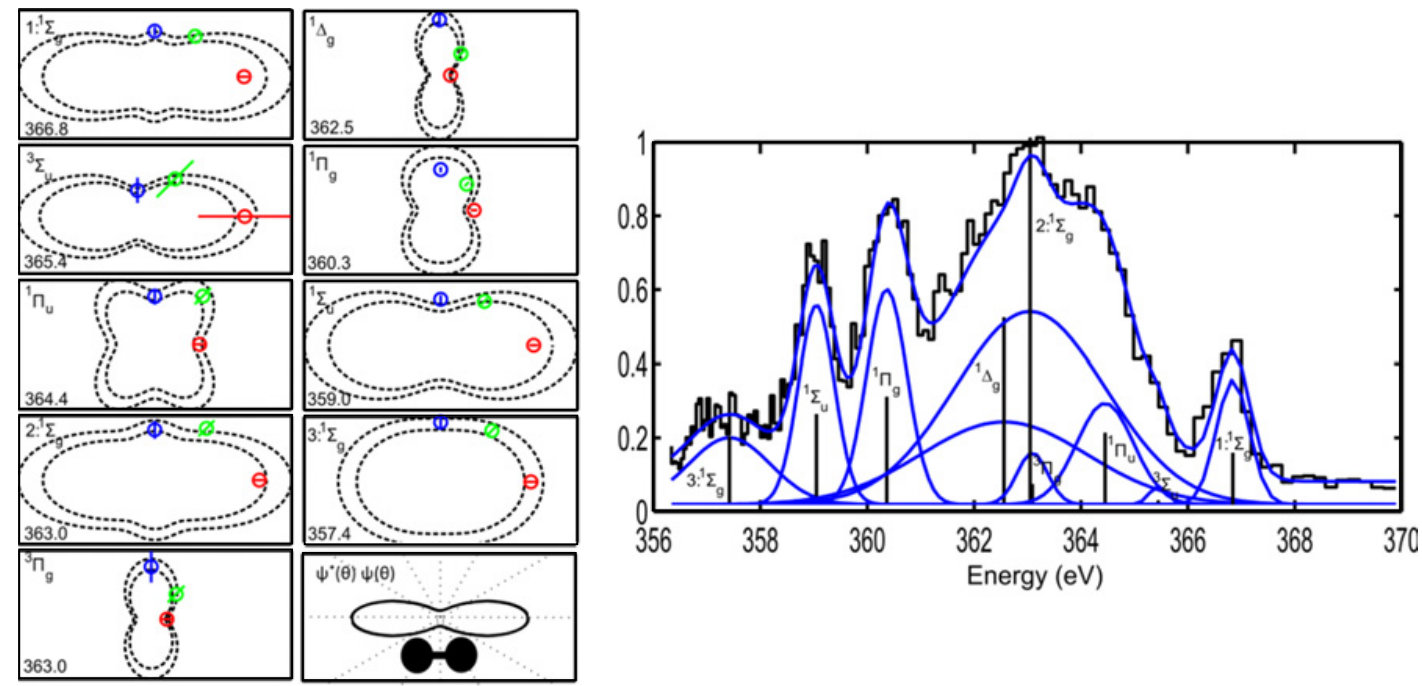

Figure 5. Left: The Auger angular distribution in $\mathrm{N}_{2}$, from LCLS measurements [9]. The dashed curve (black) shows the calculation convolved with the measured molecular ensemble distribution. Each panel is labelled by the central energy of the Auger feature (bottom-left corner) and the assigned final state (upper-left corner), in units of $\mathrm{eV}$. The states are labeled as follows: (1) $(3 \sigma g)^{-2} X$-state, (2) $(1 \pi u)^{-2},(3)(2 \sigma u)^{-2}$. Right: $\mathrm{N}_{2}$ Auger spectrum for unaligned ensemble of molecules. The curves below the composite spectrum indicate each feature's contribution to the total spectrum and are labeled next to the peak. The states in the spectrum on the right are labeled as follows: (1) $\left(3 \sigma_{g}\right)^{-2} X$-state, $(2)\left(1 \pi_{u}\right)^{-2},(3)\left(2 \sigma_{u}\right)^{-2}[9]$.

\subsection{Auger relaxation processes viewed in the molecular frame}

The ultrafast time structure of LCLS makes it possible to utilize implusive alignment techniques to view photoionization and Auger relaxation in the molecular frame. An ensemble of molecules with sufficient alignment to perform such studies can be produced through a sequential process involving initial supersonic cooling followed by laser Raman excitation. First molecules are injected into the $\mathrm{x}$ ray interaction region via a supersonic expansion, so that they are rotationally cooled to approximately $20 \mathrm{~K}$, but randomly oriented. Then an impulse from an $800 \mathrm{~nm} 100 \mathrm{fs}$ laser redistributes the rotational population into a coherent wavepacket state, which comes into maximum alignment after approximately $400 \mathrm{fs}$, and then again at intervals of $8.4 \mathrm{ps}$.

When these studies were performed the LCLS was operated in a mode with only $20 \mathrm{pC}$ of electron bunch charge, so that it produced its shortest pulses, on the order of $10 \mathrm{fs}$ or less. Auger relaxation follows within only a few fs, so that the molecular gas is effectively frozen in a state of maximum alignment. Then the Auger electron spectrum and angular distribution can be collected in the reference frame of the oriented molecules. This was the first experiment that was capable of measuring the angular distribution of Auger relaxation to the metastable states of the molecular dication. Previous experiments have used a reaction microscope technique, where the orientation is inferred from the direction of the dissociation of atomic fragments; but many states of the dication are metastable and dissociation does not occur promptly, so a reaction microscope cannot be used. In Figure 5 the results of these studies are compared with calculations of the angular distributions.

\section{CONCLUSION}

The AMO program at LCLS continues to explore the new opportunities in atomic science of high field ultrafast coherent x-ray radiation. Recent experiments include the first observation of an atomic laser 
EPJ Web of Conferences

pumped by an x-ray laser [14], and searches for nonlinear two-photon processes and coherent Rabi flopping $[15,16]$. As the quality of the laser radiation improves in power, coherence, and bandwidth, more investigations of $\mathrm{x}$-ray quantum optical phenomena will become possible.

\section{References}

[1] P. Emma, R. Akre, J. Arthur, R. Bionta, C. Bostedt, J. Bozek, A. Brachmann, P. Bucksbaum, R. Coffee, F.-J. Decker, Y. Ding, D. Dowell, S. Edstrom, A. Fisher, J. Frisch, S. Gilevich, J. Hastings, G. Hays, P. Hering, Z. Huang, R. Iverson, H. Loos, M. Messerschmidt, A. Miahnahri, S. Moeller, H.-D. Nuhn, G. Pile, D. Ratner, J. Rzepiela, D. Schultz, T. Smith, P. Stefan, H. Tompkins, J. Turner, J. Welch, W. White, J. Wu, G. Yocky, and J. Galayda, NATURE PHOTONICS 4 (2010) 641.

[2] Y. Ding, Z. Huang, D. Ratner, P. Bucksbaum, and H. Merdji, Physical Review Special Topics Accelerators and Beams 12 (2009) 060703.

[3] J. Amann, W. Berg, V. Blank, F. J. Decker, Y. Ding, P. Emma, Y. Feng, J. Frisch, D. Fritz, J. Hastings, Z. Huang, J. Krzywinski, R. Lindberg, H. Loos, A. Lutman, H. D. Nuhn, D. Ratner, J. Rzepiela, D. Shu, Y. Shvyd'ko, S. Spampinati, S. Stoupin, S. Terentyev, E. Trakhtenberg, D. Walz, J. Welch, J. Wu, A. Zholents, and D. Zhu, NATURE PHOTONICS 6 (2012) 693.

[4] J. P. Cryan, J. M. Glownia, J. Andreasson, A. Belkacem, N. Berrah, C. I. Blaga, C. Bostedt, J. Bozek, C. Buth, L. F. DiMauro, L. Fang, O. Gessner, M. Guehr, J. Hajdu, M. P. Hertlein, M. Hoener, O. Kornilov, J. P. Marangos, A. M. March, B. K. McFarland, H. Merdji, V. S. Petrović, C. Raman, D. Ray, D. Reis, F. Tarantelli, M. Trigo, J. L. White, W. White, L. Young, P. H. Bucksbaum, and R. N. Coffee, Phys. Rev. Lett. 105 (2010) 083004.

[5] J. M. Glownia, J. Cryan, J. Andreasson, A. Belkacem, N. Berrah, C. I. Blaga, C. Bostedt, J. Bozek, L. F. DiMauro, L. Fang, J. Frisch, O. Gessner, M. Gühr, J. Hajdu, M. P. Hertlein, M. Hoener, G. Huang, O. Kornilov, J. P. Marangos, A. M. March, B. K. McFarland, H. Merdji, V. S. Petrovic, C. Raman, D. Ray, D. A. Reis, M. Trigo, J. L. White, W. White, R. Wilcox, L. Young, R. N. Coffee, and P. H. Bucksbaum, Opt. Express 18 (2010) 17620.

[6] L. Fang, M. Hoener, O. Gessner, F. Tarantelli, S. T. Pratt, O. Kornilov, C. Buth, M. Gühr, E. P. Kanter, C. Bostedt, J. D. Bozek, P. H. Bucksbaum, M. Chen, R. Coffee, J. Cryan, M. Glownia, E. Kukk, S. R. Leone, and N. Berrah, Phys. Rev. Lett. 105 (2010) 083005.

[7] M. Hoener, L. Fang, O. Kornilov, O. Gessner, S. T. Pratt, M. Gühr, E. P. Kanter, C. Blaga, C. Bostedt, J. D. Bozek, P. H. Bucksbaum, C. Buth, M. Chen, R. Coffee, J. Cryan, L. DiMauro, M. Glownia, E. Hosler, E. Kukk, S. R. Leone, B. McFarland, M. Messerschmidt, B. Murphy, V. Petrovic, D. Rolles, and N. Berrah, Phys. Rev. Lett. 104 (2010) 253002.

[8] L. Young, E. P. Kanter, B. Krässig, Y. Li, A. M. March, S. T. Pratt, R. Santra, S. H. Southworth, N. Rohringer, L. F. DiMauro, G. Doumy, C. A. Roedig, N. Berrah, L. Fang, M. Hoener, P. H. Bucksbaum, J. P. Cryan, S. Ghimire, J. M. Glownia, D. A. Reis, J. D. Bozek, C. Bostedt, and M. Messerschmidt, Nature 466 (2010) 56.

[9] J. P. Cryan, J. M. Glownia, J. Andreasson, A. Belkacem, N. Berrah, C. I. Blaga, C. Bostedt, J. Bozek, N. A. Cherepkov, L. F. DiMauro, L. Fang, O. Gessner, M. Gühr, J. Hajdu, M. P. Hertlein, M. Hoener, O. Kornilov, J. P. Marangos, A. M. March, B. K. McFarland, H. Merdji, M. Messerschmidt, V. S. Petrović, C. Raman, D. Ray, D. A. Reis, S. K. Semenov, M. Trigo, J. L. White, W. White, L. Young, P. H. Bucksbaum, and R. N. Coffee, Journal of Physics B: Atomic, Molecular and Optical Physics 45 (2012) 055601.

[10] J. M. Glownia, in Applied Physics, Vol. Ph.D., Stanford University, 2012.

[11] J. P. Cryan, in Physics, Vol. Ph.D., Stanford University, 2012.

[12] J. J. Yeh and I. Lindau, Atomic Data and Nuclear Data Tables 32 (1985) 1.

[13] N. Berrah, J. Bozek, J. T. Costello, S. Düsterer, L. Fang, J. Feldhaus, H. Fukuzawa, M. Hoener, Y. H. Jiang, P. Johnsson, E. T. Kennedy, M. Meyer, R. Moshammer, P. Radcliffe, M. Richter, 
A. Rouzée, A. Rudenko, A. A. Sorokin, K. Tiedtke, K. Ueda, J. Ullrich, and M. J. J. Vrakking, Journal of Modern Optics 57 (2010) 1015.

[14] N. Rohringer, D. Ryan, R. A. London, M. Purvis, F. Albert, J. Dunn, J. D. Bozek, C. Bostedt, A. Graf, R. Hill, S. P. Hau-Riege, and J. J. Rocca, Nature 481 (2012) 488.

[15] G. Doumy, C. Roedig, S.-K. Son, C. I. Blaga, A. D. DiChiara, R. Santra, N. Berrah, C. Bostedt, J. D. Bozek, P. H. Bucksbaum, J. P. Cryan, L. Fang, S. Ghimire, J. M. Glownia, M. Hoener, E. P. Kanter, B. Krässig, M. Kuebel, M. Messerschmidt, G. G. Paulus, D. A. Reis, N. Rohringer, L. Young, P. Agostini, and L. F. DiMauro, Phys. Rev. Lett. 106 (2011) 083002.

[16] E. P. Kanter, B. Krässig, Y. Li, A. M. March, P. Ho, N. Rohringer, R. Santra, S. H. Southworth, L. F. DiMauro, G. Doumy, C. A. Roedig, N. Berrah, L. Fang, M. Hoener, P. H. Bucksbaum, S. Ghimire, D. A. Reis, J. D. Bozek, C. Bostedt, M. Messerschmidt, and L. Young, Physical Review Letters 107 (2011) 233001. 